\title{
An ad-hoc fretting wear tribotester design for thin steel wires
}

\author{
Iñigo Llavori ${ }^{1, *}$, Alaitz Zabala ${ }^{1}$, Mikel Aingeru Urchegui $^{2}$, Wilson Tato ${ }^{1}$, Andrea Aginagalde ${ }^{1}$, Iñaki Garate ${ }^{1}$, and Xabier \\ Gómez ${ }^{1}$
}

${ }^{1}$ Surface Technologies, Mondragon University, Loramendi 4, 20500 Arrasate-Mondragón, Spain

${ }^{2}$ Orona EIC, Orona Ideo, Jauregi bidea s/n, 20120, Hernani, Gipuzkoa, Spain

\begin{abstract}
Steel wire ropes experience fretting wear damage when the rope runs over a sheave promoting an oscillatory motion between the wires. Consequently, wear scars appear between the contacting wires leading to an increase of the stress field and the following rupture of the wires due to fatigue. That is why the understanding and prediction of the fretting wear phenomena of thin wires is fundamental in order to improve the performance of steel wire ropes. The present research deals with the design of an ad-hoc fretting wear test machine for thin wires. The test apparatus is designed for testing thin wires with a maximum diameter of $1.0 \mathrm{~mm}$, at slip amplitudes ranging from 5 to $300 \mu \mathrm{m}$, crossing angle between $0-90^{\circ}$, and contacting force ranging from 0,5 to $5 \mathrm{~N}$. The working principle of displacement amplitude and contacting force as well as the crossing angle between the wires are described. Preliminary studies for understanding the fretting wear characteristics are presented, analysing $0.45 \mathrm{~mm}$ diameter cold-drawn eutectoid carbon steel $(0.8 \% \mathrm{C})$ wires (tensile strength higher than $3000 \mathrm{MPa})$.
\end{abstract}

\section{Introduction}

Many mechanical components such as, bearing housings, flexible couplings and spines or wire ropes are simultaneously subjected to a fretting wear and fatigue damage. Fretting phenomenon arises when two bodies are in contact subjected to relative movement of small amplitude, producing damage to the contact surface [1]. Depending on the magnitude of stresses, fretting phenomenon can cause catastrophic failure of such mechanical components.

Steel wire ropes exhibit high axial strength and bending flexibility. These properties are largely dependent on their construction and the manufacturing process of the wires. Despite their outstanding mechanical properties, steel wire ropes are prone to fretting wear damage due to the oscillatory motion between wires. The wear scars appearing at the contact interface increase the stress fields, leading to the rupture of the wires due to fatigue, and eventually the failure of the rope. The correct selection of wire material and rope configuration is therefore crucial in order to increase the rope life, which demands the characterization of the steel wire behaviour.

Steel wire's fretting wear behaviour characterization requires the use of a fretting tribometer. A tribometer is a test machine capable of measuring tribological parameters between surfaces in contact, such as friction force, coefficient of friction or wear volume. This work presents the design of the developed ad-hoc tribotester, designed for the characterization of thin steel wires under highly stable displacement strokes and contact forces.

\section{Development of a fretting wear tribotester}

\subsection{Design requirements}

The main challenge on the design of a fretting test lies on the precise control of the displacement amplitude and the contact force. On the one hand, fretting stroke is established between 0 and $300 \mu \mathrm{m}$ [1]. On the other hand, previous works identified that premature rupture of the wires occurred for a contact force higher than $3 \mathrm{~N}$ $[2,3]$. Furthermore, it should be considered that the construction of a rope presents multiple wire contacts at different angles. Therefore, the test rig should be able to reproduce those crossing angles. Accordingly, the design requirements are defined as follows:

- $\quad$ Displacement amplitude ranging from 5 to $300 \mu \mathrm{m}$.

- Crossing angle ranging from 0 to 90 degrees.

- Contact force ranging from 0 to $5 \mathrm{~N}$

\subsection{Displacement amplitude module design}

Servo-hydraulic [4], piezo-electric [5] or electromechanical actuators $[6,7]$ can be used to generate a short cyclic displacement. The main characteristics of each actuator can be summarized as follows:

- Servo-hydraulic actuators are generally used in high demanding machines, since they provide a very stiff driving system with a precise control of the stroke.

Corresponding author: illavori@mondragon.edu 
- Piezo-electric actuators are ideal for a low mass, high frequency and short stroke applications. Fatigue life of the piezo-electric actuator is dependent on the displacement amplitude.

- Electro-mechanical systems converts the rotational motion into linear motion. They provide a simple and cost effective solution.

Fig. 1 shows the camshaft mechanism designed for the tribotester displacement system. A variable eccentric connected to an electro-mechanical motor converts the rotational motion into a reciprocating motion. Aditionally, two oscillating rods connected in series allow to reduce the displacement stroke into a highly stable short movement.

Guide 2

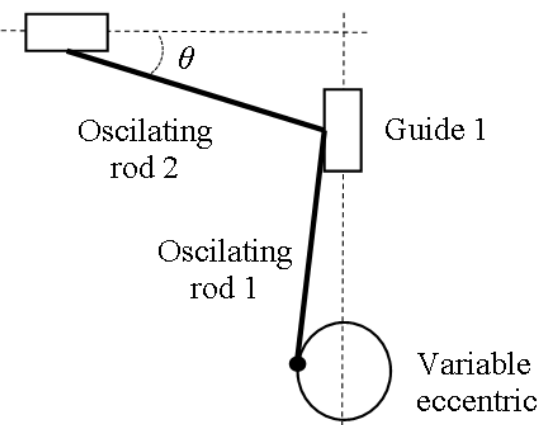

Fig. 1. The camshaft mechanism designed for the tribotester displacement.

Note that the displacement reduction is dependent on the angle $\theta$ : lower values of cyclic amplitude can be obtained with lower angles. The actual amplitude of cyclic displacement can be calculated from:

$$
\delta=l_{\text {or } 2}\left(\cos \theta_{1}-\cos \theta_{2}\right)
$$

where $\delta$ is the displacement amplitude, $l_{\text {or } 2}$ is the oscillating rod 2 length and $\theta_{1}$ and $\theta_{2}$ are the maximum and minimum angles obtained with the variable eccentric.

\subsection{Contact force and wire fixation module design}

Contact force can be applied by dead weight system $[6,7,8]$, linear actuators [9] or a mechanical screw type actuator with an adjustable spring [10]. It should be noted that low mass loading systems such as spring or pneumatic actuators produce less severe conditions than dead weight loading systems, since the latter gives rise to shock loading.

The adopted solution in the tribotester is therefore the screw type actuator with an adjustable spring. This mechanical system in combination with a load cell allows to measure in real-time the contact force, avoiding the necessity of doing the hypothesis of a constant normal force.

Low contact forces between thin steel wires generate high stresses in the contact interface. Therefore, the need for reliable low force measurement is critical in the present application. Generally, contact and friction force measurements are decoupled, i.e. multiple uniaxial sensors are used to monitor each force $[6,7,8,11]$. This concept is simple in design, nonetheless it presents the following disadvantages:

- $\quad$ Large (space limitation).

- Misalignment and angle errors due to installation problems.

Lack of stiffness which leads to linearity errors.

Difficult compensation for the crosstalk effect.

Fig. 2 shows the multiaxial load cell capable of monitoring forces in three axis adopted in the designed tribotester and the schematic of the configuration. The benefits of the multiaxial sensors are listed below:

- Compact design.

- $\quad$ Same reference point for all force components.

- $\quad$ Easy compensation for the crosstalk effect.
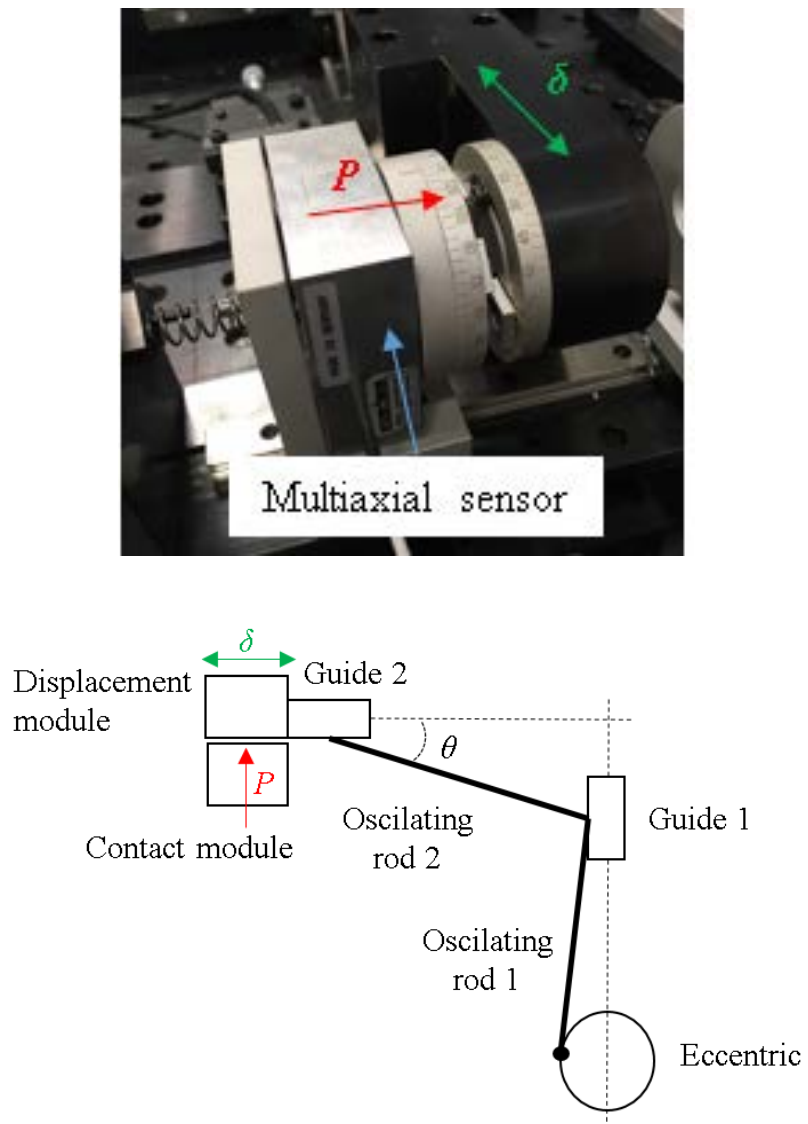

Fig. 2. Upper figure, Multiaxial load cell capable of monitoring forces in three axis adopted in the designed tribotester. (Lower figure, Schematic of the tribotester configuration.

Fig. 3 shows the developed wire fixation system, which avoids non desired movements. As can be noted in the figure, the wire presents a toroid shape, being the radius of the support considerably superior comparing to the wire diameter. Therefore, the contact achieved between the two wires can be considered a cross cylinder configuration. It should be noted that the fixing system allows to locate the wire at different angles, thus being possible to simulate different contacts present in ropes. 


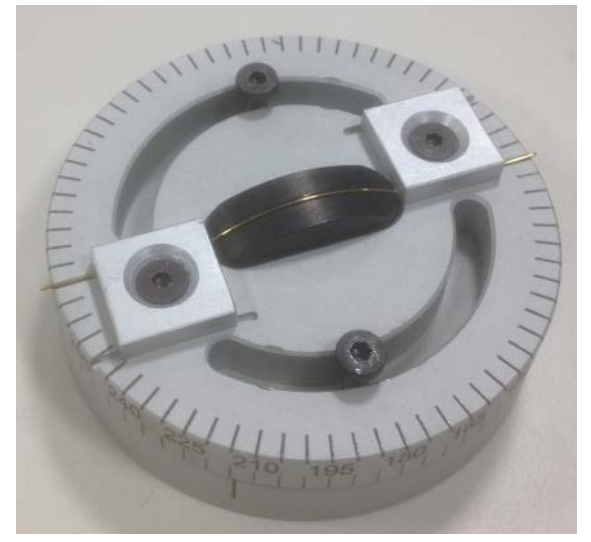

Fig. 3. Thin wires fixation system.

\section{Verification tests}

Fretting loops show the relationship between slip displacement and friction force along a fretting cycle. The shape of the fretting loop gives information on the fretting regime: where an elliptic shape is related with partial slip regime (Figure 4 (a)), and shifted rectangle is associated with gross slip regime (Figure 4 (b)). Furthermore, the area of the fretting loop can be used to determine the the coefficient of friction and wear.

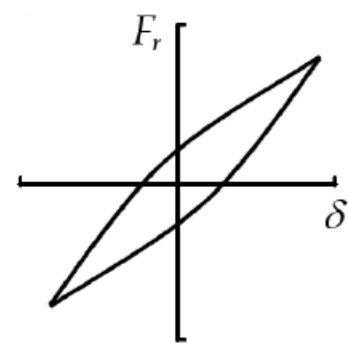

(a)

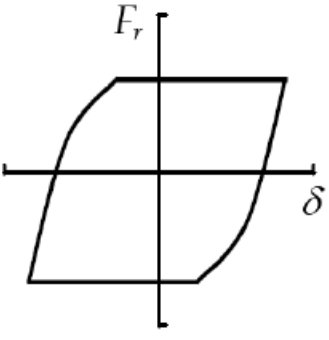

(b)
Fig. 4: Fretting loop shape. (a) partial slip regime; (b) gross slip regime [12].

Preliminary studies for understanding the fretting wear characteristics were conducted on $0.45 \mathrm{~mm}$ diameter cold-drawn eutectoid carbon steel $(0.8 \%$ C) with a tensile strength higher than $3000 \mathrm{MPa}$. Fig. 5 and Fig. 6 show the unfiltered measurements of the contact $(\mathrm{P})$ and friction (Q) forces and the monitored fretting loop respectively. The obtained results show a highly stable curves with very low signal to noise ratio, thus verifying the correct behaviour of the developed tribotester. It is expected that further analysis using the test rig will allow a better understanding of wear behaviour in different contact configurations.

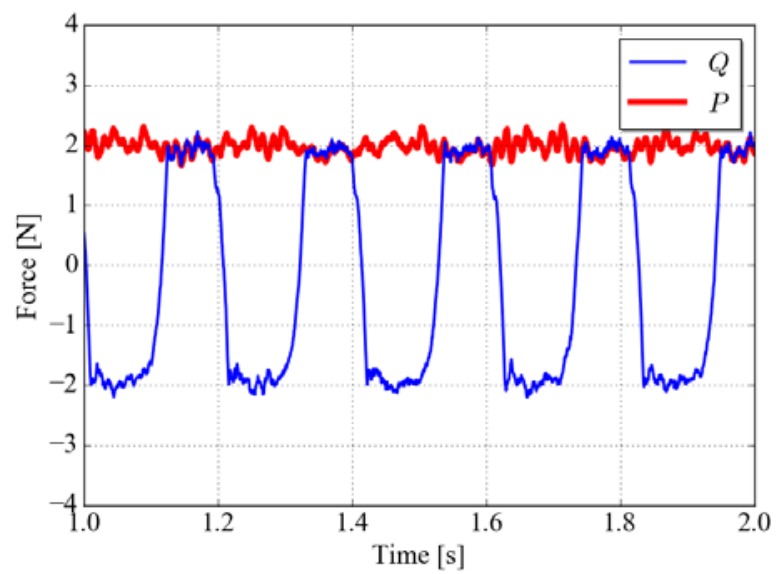

Fig. 5. Measured data. Contact $(\mathrm{P})$ and friction $(\mathrm{Q})$ forces.

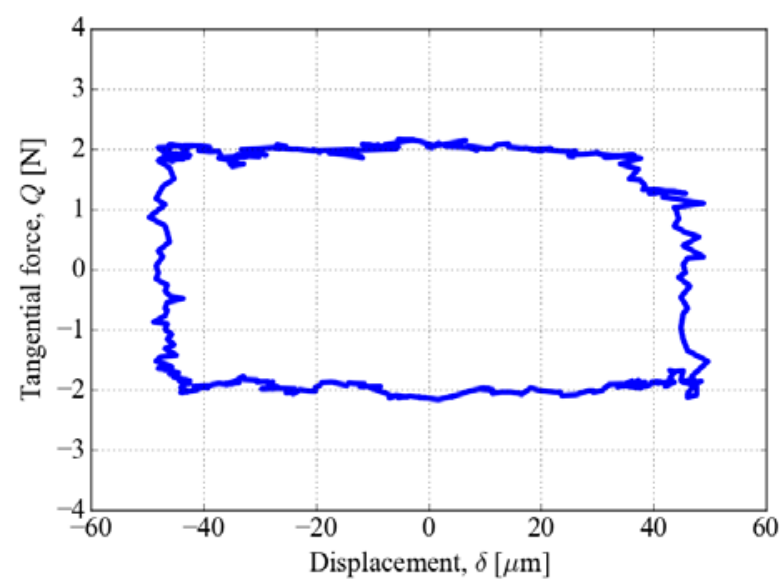

Fig. 6. Measured data. Fretting loop in gross slip regime.

\section{Conclusions}

A fretting wear tribotester for thin steel wires was developed. Highly stable data were measured validating the designed test rig. It is expected that further analysis using the test rig will allow a better understanding of fretting-wear behaviour of thin wires in different contact configurations, which may contribute to foster advancements in the design of better performing ropes.

\section{Acknowledgment}

The authors gratefully acknowledge the financial support given by the Basque Government under the "MEDECA ELKARTEK"

\section{References}

1. R.B. Waterhouse, Wear, 100: pp. 107-118 (1984).

2. A. Cruzado, M. Hartelt, R. Wäsche, M.A. Urchegui, X. Gómez, Wear, 268: pp. 1409-1416 (2010).

3. A. Cruzado, M. Hartelt, R. Wäsche, M.A. Urchegui, X. Gómez, Wear, 273: pp. 60-69 (2011). 
4. S. Fouvry, Ph. Kapsa, L. Vincent, Wear, 247: pp. 41-54 (2001).

5. E. Marui, H. Endo, N. Hasegawa, H. Mizuno, Wear, 214: pp. 221-230 (1998).

6. D. Klaffke, Wear, 104; pp. 337-343 (1985).

7. R. Ramesh, R. Gnanamoorthy, Materials \& Design, 27: pp. 141-146 (2006).

8. D. Wang, D. Zhang, S. Ge, Tribology International, 52: pp. 178-189 (2012).

9. H. Mohrbacher, J.P. Celis, J.R. Roos, Tribology International, 28: PP. 269-278C (1995).

10. B.U. Wittkowsky, P.R. Birch, J. Dominguez, S. Suresh. FFEMS, 22: pp. 307-320 (1999).

11. J. Vázquez, Phd Thesis, Universidad de Sevilla. (2009).

12. L. Vincent, Y. Berthier, M. Godet, ASTM, pp. 3348 (1992). 